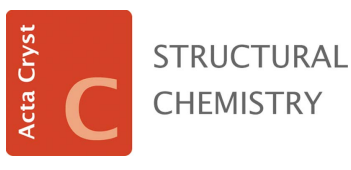

ISSN 2053-2296 Keywords: book review; periodic table; chem-
istry of life; evolution; geochemistry; teaching

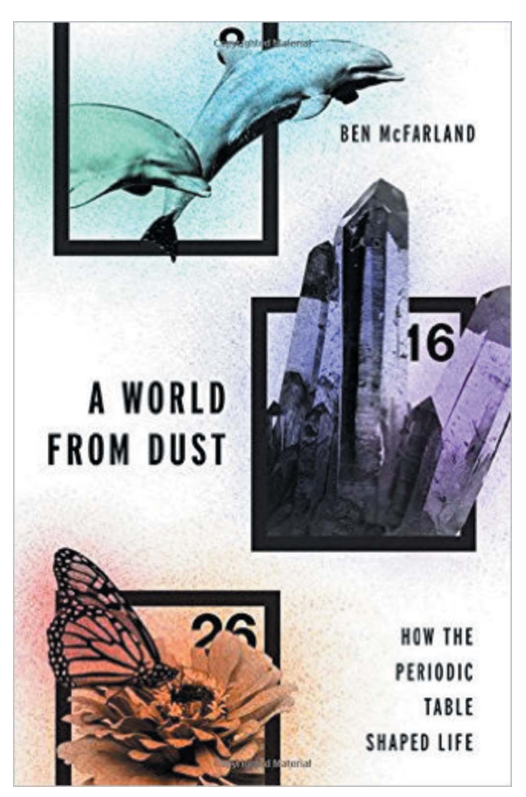

\section{A World From Dust. How the Periodic Table Shaped Life. By Ben McFarland. Oxford University Press, 2016. Hardback, Pp. XIV+333. Price GBP 22.99, USD 34.95. ISBN 9780190275013}

\author{
Marc Hebrant*
}

Université de Lorraine, LCPME, UMR 7564, Villiers-lès-Nancy, F-54601, France, and CNRS, LCPME, UMR 7564 Villiers-lès-Nancy, F-54601, France. *Correspondence e-mail: marc.hebrant@univ-lorraine.fr

'A world from dust' is not the most explicit title for a scientific book; indeed, upon hearing it, one can easily imagine its referring to a novel or a fictional tragedy. In fact, the book seems to be exactly on the borderline between a scientific work and a comic strip, explaining chemistry and science. This feeling is reinforced by the illustrations, which are sometimes very simplistic; but the reader readily understands that this book contains genuine science, heavily loaded with knowledge and captivating content.

The author proposes that you 'put on your imaginary chemical glasses' to discover how chemistry governs life and thus chemical evolution, even if you are not at all familiar with chemical formalism, which has been deliberately omitted from this book.

The number of subjects raised and the breadth of the scientific scope covered by the book are huge: for example, the role of phosphorus and its partner magnesium, illustrating the necessity of ionic balance for life (starting with the story of Mono Lake, a lake containing a high content of arsenic, and the diffusion of erroneous information), the chemistry in a cell (starting with a modern sculpture in the north of Scotland to illustrate the exchanges of a cell with the exterior and the means by which they occur), the role of liquid in life (Titan would be the most probable place after earth for life to develop), the beauty of the periodic table (starting from numerology to introduce the necessity of chemical quantum mechanics) and, of course, 'the seven chemical clues for the first life'. The diversity and number of different subjects are such that it would be almost impossible to provide a summary.

Most of the examples given being very original, there is a lot to learn from this book. In his willingness to explain science without equations, the author has created a lot of images and made a lot of analogies which presumably could be criticized by the most rigorous scientists, but will constitute a gold mine for those who, almost without a prerequisite for maths, aim to address the origin of life and the chemical reasons for evolution. Teachers in chemistry or geochemistry searching for original examples to illustrate their lessons will find a lot in this book, including solidarity ('demos always work great until the class is watching' is so true!!!). Moreover, the author addresses the various subjects from an unprecedented angle that could inspire many colleagues.

The main flaw of this book is the absence of a detailed table of contents. This omission is exacerbated by the fact that the links between the epithets listed on the first page of a chapter and the paragraph titles included in the chapter are not obvious; and anyway, neither the paragraph titles nor the epithets give a clear idea of the content (but considering the very original way the story is told, one may wonder 'how could this be the case?'). The index might be useful to some, but the truth is that the only way to read this book is as one would a novel, i.e. sequentially. There is no way to find a specific part of the story, and one who would not enter into the chemical universe of the author from the beginning (i.e. the first line of the first chapter) would have difficulties penetrating it thereafter. For instance, in the $6^{\text {th }}$ chapter, entitled 'wheels within wheels,' there are multiple references to all the preceding chapters, from the Mono Lake story (chapter 1) to the chemistry of the early earth (chapter 5).

In my opinion, the original approach of the author in describing the links between chemical properties, natural abundances of elements, and the origin of life and its evolution deserves the readers' dedication of the necessary time to read this book from the beginning. Moreover, it is very well documented, with 43 pages of detailed bibliographic references supporting the preceding chapters, which allow the reader to explore in depth the subjects discussed. 